\title{
A study on an electromagnetic shield with carbon filler
}

\author{
N. M. Legkiy ${ }^{*}$ \\ MIREA - Russian Technological University, Vernadskogo Av., 78, Moscow 119454, Russia
}

\begin{abstract}
This article presents protective properties of an electromagnetic metal shield with the carbon black filler, as well compares its characteristics with ones that regular metal protective shield has. There are three methods to compare, namely the first is calculation, next one is simulation in CST software, and finally are experimental methods. The study results obtained with all three methods are the same. Therefore, after multiple comparison of the results obtained with two types of shields, we can say that the electromagnetic shield with the carbon filler has better shielding characteristics.
\end{abstract}

\section{Introduction}

A need arises to protect the workers against the electromagnetic fields when they work with radio-emitting equipment and other equipment such as radio transmitters or power converters that generate powerful electromagnetic fields. The shielding is one of the most effective protection methods against the electromagnetic fields and the radio frequencies. A protection level largely depends on the design of the shield. There are two types of the electromagnetic shields, namely reflective (rereflective) and absorptive. Today researchers try to find inexpensive and effective ways for protection.

Besides, many studies have been devoted to research in the protection of workers against the electromagnetic fields (EMF) in the radio frequency (RF) range as well as search for the most effective protective shields, for example [1-7].

\section{Research objective}

The electromagnetic waves have various effects on different materials that are used to protect people. Here are tested some types of protective shields, including those with the carbon black filler.

Radiation exposure and measurements of the reflected signal will be done at $\mathrm{X}$ band frequency range from 8 to $12 \mathrm{GHz}$.

\footnotetext{
*Corresponding author: legki@mirea.ru
} 
In terms of design, the protective shield has a shape of rectangular parallelepiped with a square cavity, including its side has $156 \mathrm{~mm}$ and the depth is $4 \mathrm{~mm}$. During the measurements sample study materials will be placed in that cavity (Fig. 1).

The metal plate is produced from Perfect Electric Conductor (PEC) in accordance with the dimensions shown in Figure 1.

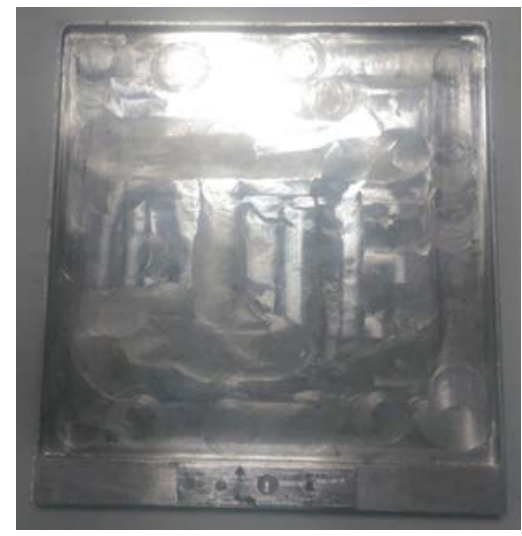

a)

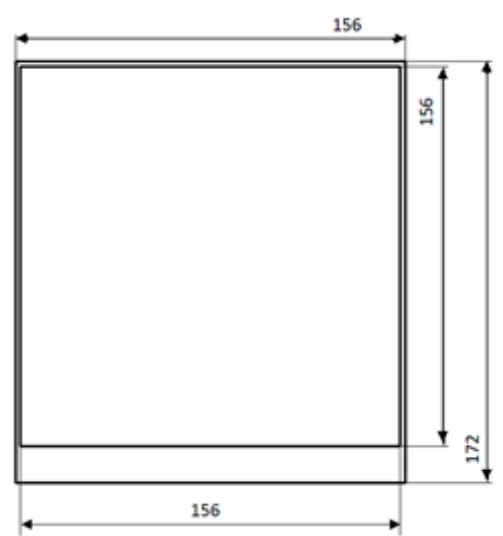

b)

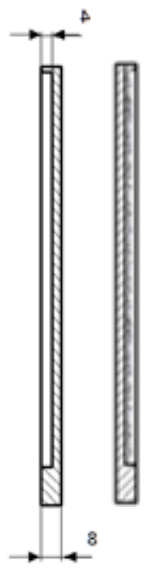

c) d)

Fig. 1. Exterior appearance (a), Dimensions of the study shield (b, c) and Sample with the filler (d).

Figure 2 shows a model of the metal shield, a CST software package was used for designing (www.cst.com).

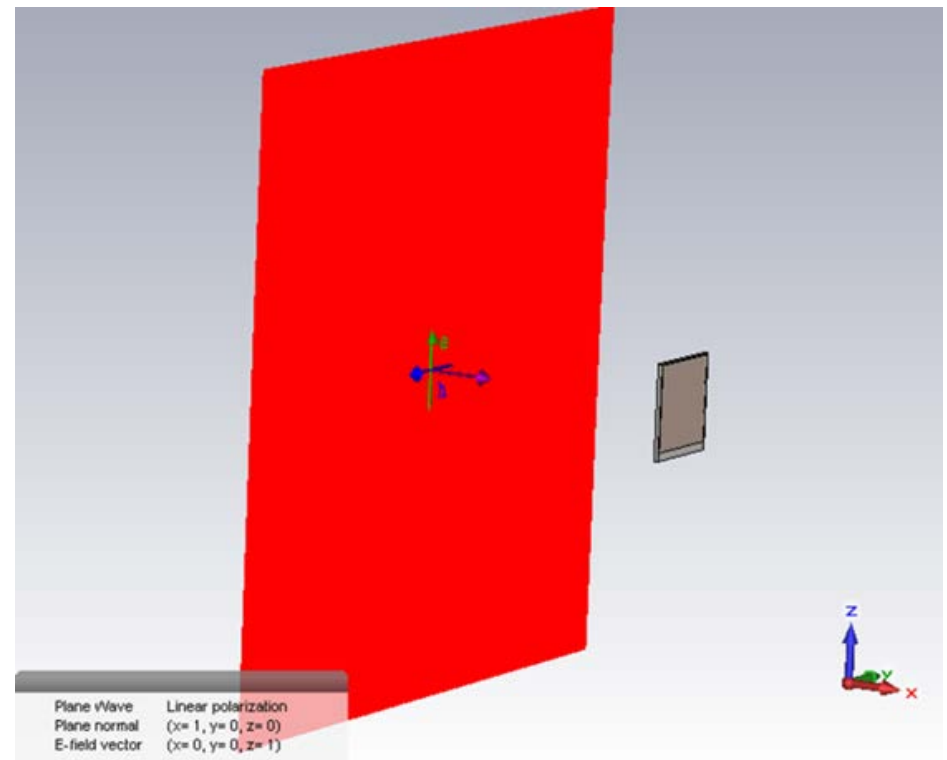

Fig. 2. General view of the model with a plane wave.

The study of protective shields will be done using a setup shown in Fig. 3. 

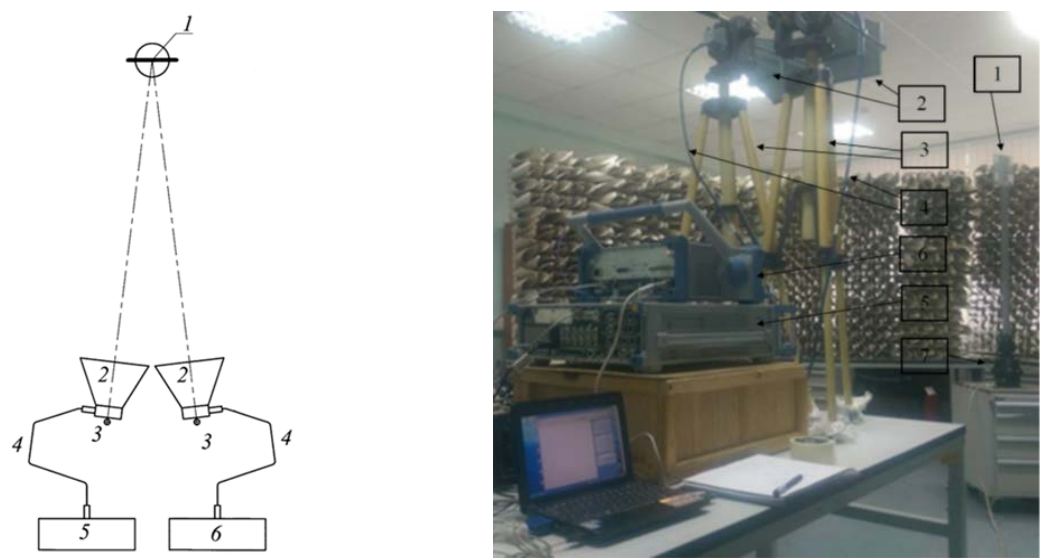

Fig. 3. Schematic diagram of the measuring setup. 1. Measured sample, 2. Measuring antenna, 3. Holder, 4. Cable, 5. Signal generator ROHDE \& SCHWARZ SMF 100A, 6. Spectrum analyzer ROHDE \& SCHWARZ FSL-18, 7. Rotary drive.

Used fillers:

- Carbon black.

Carbon black is a form of thermo-oxidative or thermal decomposition of hydrocarbons in the gas phase.

To determine the radio-reflecting and radio-absorbing properties of shields with different fillers, we compare the signal power that is reflected from the "dummy" plate and the plate with different fillers using a range of frequencies at the beginning and end of the $\mathrm{X}$ band.

\section{Calculation methods of protection against electromagnetic fields of radio frequencies}

Generally, the shielding coefficient (attenuation) of the electromagnetic fields of radio frequencies is defined as

$$
\begin{aligned}
& \mathrm{A}_{\mathrm{E}}=20 \lg \left(\mathrm{E}_{0} / \mathrm{E}_{\mathrm{A}}\right), \\
& \mathrm{A}_{\mathrm{H}}=20 \lg \left(\mathrm{H}_{0} / \mathrm{H}_{\mathrm{A}}\right),
\end{aligned}
$$

Where $A_{E}$ is the shielding coefficient (attenuation) for the electric component of the electromagnetic field, $\mathrm{dB}$

$\mathrm{A}_{\mathrm{H}}$ is the shielding coefficient (attenuation) for the magnetic component of the electromagnetic field, $\mathrm{dB}$

$\mathrm{E}_{0}$ is the electric intensity of the electromagnetic field at the measurement point when the shield is absent, $\mathrm{V} / \mathrm{m}$

$\mathrm{E}_{\mathrm{A}}$ - the electric intensity of the electromagnetic field at the measurement point when the shield is present, $\mathrm{V} / \mathrm{m}$

$\mathrm{H}_{0}$ is the magnetic intensity of the electromagnetic field at the measurement point when the shield is absent, $\mathrm{A} / \mathrm{m}$

$\mathrm{H}_{\mathrm{A}}$ is the electric intensity of the electromagnetic field at the measurement point when the shield is present, $\mathrm{A} / \mathrm{m}$

The attenuation of the electromagnetic wave $K_{e}$ in the shield is defined as:

$$
K_{e}=\frac{(n+1)+\chi^{z}}{4 n} \exp \left(\frac{z \cdot \chi \cdot \omega \cdot x}{c}\right),
$$

Where $\mathrm{n}$ is the refraction coefficient of the material 
$\chi$ is the extinction coefficient of the material, which determines the velocity of wave attenuation

$\omega$ is the circular frequency of radiation $(\omega=2 \pi f)$

$x$ is the thickness of the sample

In the case, if the incident wave is normal, the reflection coefficient $\mathrm{Kv}$ of the shield is determined as:

$$
K_{V}=\frac{(n-1)^{2}+\chi^{z}}{(n+1)^{2}+\chi^{z}},
$$

Where $n$ is the refraction coefficient of the shielding material

$\chi$ is the attenuation coefficient of the shield material, which determines the velocity of wave attenuation

The value of the attenuation coefficient $\chi$ and the value of the reflection coefficient of the material $\mathrm{n}$ are determined as:

$$
\begin{aligned}
& \chi=\sqrt{\frac{\varepsilon^{\prime}-\sqrt{\varepsilon^{\prime 2}+\varepsilon^{\prime \prime 2}}}{2}}, \\
& n=\sqrt{\frac{\varepsilon^{\prime}+\sqrt{\varepsilon^{\prime 2}+\varepsilon^{\prime \prime 2}}}{2}},
\end{aligned}
$$

Where $\varepsilon^{\prime}$ is the real part of the complex dielectric permittivity of the shield $\varepsilon^{\prime \prime}$ is the imaginary part of the complex dielectric permittivity

$$
\omega=\varepsilon+i(4 \sigma / \omega)
$$

Where $\sigma$ is the electrical conductivity of the material $\omega$ is the circular frequency of radiation

In addition, the properties of the protective shield also depend on the angle at which the radio wave hits the shield.

\section{Experimental studies on the shields using $X$ band frequency range}

We are going to study the electromagnetic protective shields. We will also take measurements at the beginning $(8 \mathrm{GHz})$ and at the end $(12 \mathrm{GHz})$ of the $\mathrm{X}$ band frequency ranges.

\subsection{The metal shield}

The charts in Microsoft Excel show 3 curves: (1) A distribution that is obtained by modeling in CST software. (2) The experimentally measured power distribution. (3) The background at a given frequency. 


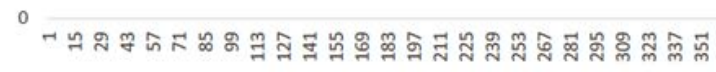

$-10$

$-20$

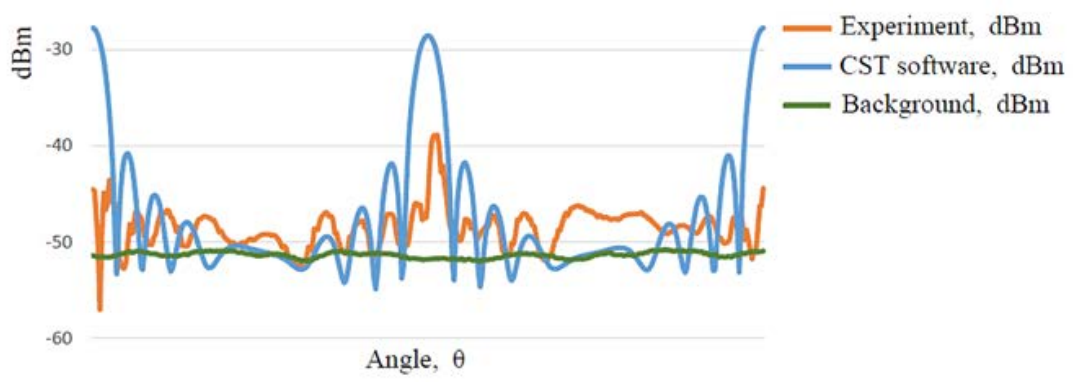

Fig. 4. The power distribution that is reflected from the plate at the $8 \mathrm{GHz}$ frequency

According to the charts in Figure 4, we can say that the reflected signal that was received by the antenna is a mixture of the background noise and the useful signal. The figure shows that the experimental values are close to theoretical ones at the frequency of $8 \mathrm{GHz}$. Considering the plate setup tolerances, peaks coincide with both width and maximum. We could perfectly distinguish a main lobe as well as the side lobes. The difference between the power of the reflected signal in the main lobe of the theoretical and experimental distributions is about 2 times of the maximum at the given frequency. This power drops because when some energy portion of the electromagnetic wave hits the shield it is converted into thermal energy. When the wave gets into conductive medium it attenuates rapidly, because in the plate occur high frequency conduction currents that heat the medium substance [8]. Besides, there are factors that have influence on that, namely wave interference, which are rereflected from objects of the environment [9], losses when the antenna receives the signals [10-12], power loss of the incident and reflected waves into space, and some other factors [9].

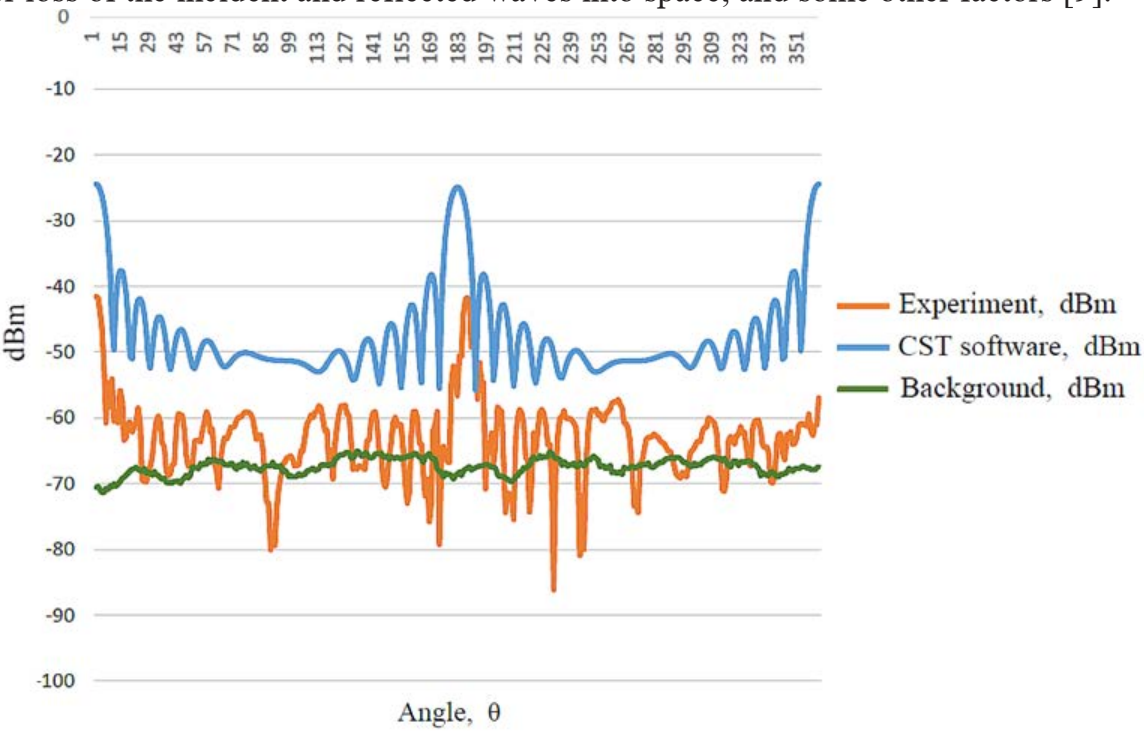

Fig. 5. The power distribution that is reflected from the plate at the $12 \mathrm{GHz}$ frequency 
Figure 5 shows that the experimental values practically coincide with the theoretical ones at the $12 \mathrm{GHz}$ frequency. The side lobes are also clearly distinguishable just like in the power distribution at the $8 \mathrm{GHz}$ frequency. The difference between the reflected signal in the main lobe of the theoretical and experimental distributions is practically absent at a given frequency, namely it is within $1 \ldots 2 \mathrm{~dB}$. If we compare with the previous frequency the losses are smaller because there is higher frequency, and this means that a wavelength is shorter as well.

\subsection{Measurement of the reflected signal using the carbon black as the filler}

Figure 6 shows that the plate with the carbon black makes the main peak almost drop down to the level of the side lobes at the $8 \mathrm{GHz}$ frequency.

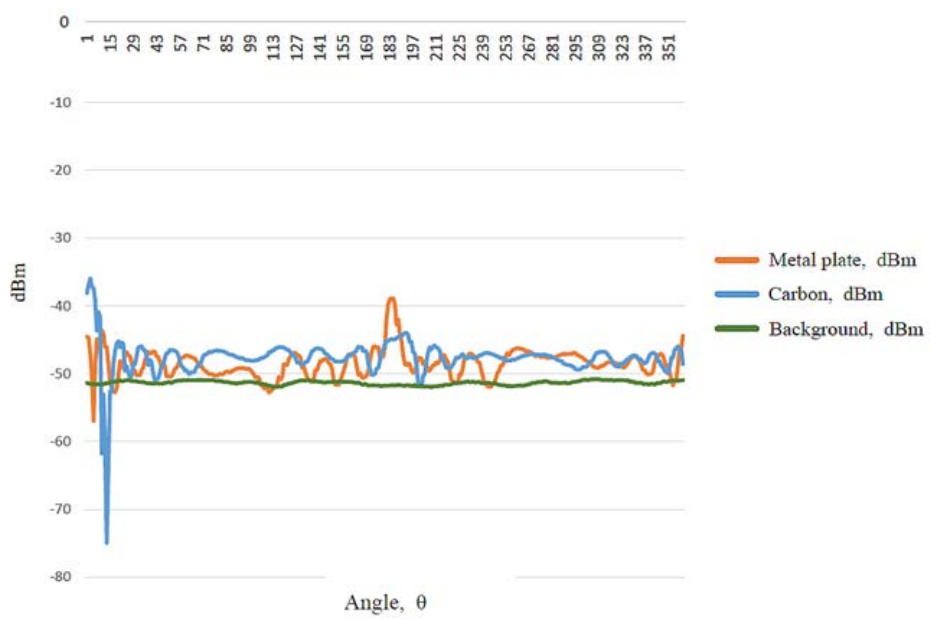

Fig. 6. The power distribution that is reflected from the plate using the carbon black at the $8 \mathrm{GHz}$ frequency

The experiment at the $12 \mathrm{GHz}$ frequency has the same effect as at the $8 \mathrm{GHz}$ (Fig. 7). In addition, a dip occurs at the center of the peak, which means there is not the signal reflection, and low levels of reflected power let us to draw a conclusion that these shields largely absorb the energy rather than reflect it.

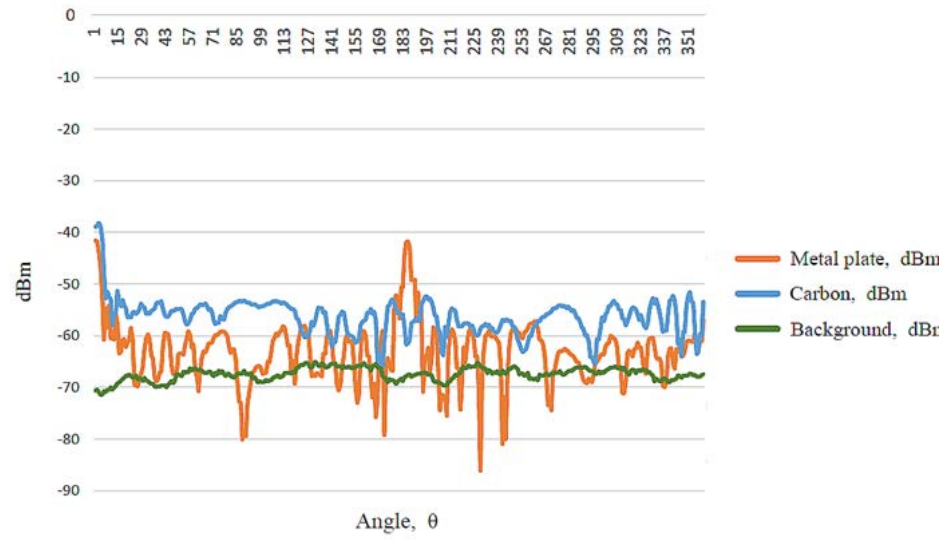

Fig. 7. The power distribution that is reflected from the plate using the carbon black at the $12 \mathrm{GHz}$ frequency 


\section{Conclusion}

Analyzing the results from the experimental measurements on the reflected signals at X band frequency ranges and comparing them with computer simulation we can see that they coincide with the existing tolerances, losses and interferences. This suggests that the experiment was done correctly. Therefore, the presented technique can be used to measure different types of reflective shields.

In addition to that, there were discovered some distinctive distribution elements of the reflective shield characteristics, according to which it could be argued that the presence of the main peak and the side lobes make the protective shields as the reflective ones despite of any interference and other material. During the experiment, it was also discovered that the dielectrics are radiotransparent and have no influence on the absorbing properties of shields.

Analyzing the results, we can confidently state that carbon black is a radiation-absorbent material. The tolerances that were obtained from the experiment could be caused by the uneven distribution of carbon black on the metal plate surface.

\section{References}

1. S. Fahy, C. Kittel, S.G. Louie Electromagnetic screening by metals American Journal of Physics, November 1988, 56:11(11),

2. F.D. Iosif, M.A. Costea Ecrane electromagnetice multistrat pentru aparatura electronica / Electrotehnica, Electronica, Automatica, 2014, 62 (3), 96.

3. Modelling and testing the electromagnetic near field shielding effectiveness achieved by woven fabrics with conductive yarns IR Rădulescu, E Visileanu, I Pătru, L Surdu, M Costea, V Voicu Industria Textila 69 (3), 169-176

4. Pozar, D.M. Microwave engineering / John Wiley \& Sons, 2005.

5. Boiprav O.V., Bogush N.V., Lynkou L.M. Radioshielding properties of composites based on titanium dioxide. Doklady BGUIR. 2017, Vol.110 (8), pp.67-72.(in Russia)

6. Abasov R.K. The use of carbon materials in the shielding of electromagnetic fields / Polytechnic youth magazine. 2016. No. 5. P.1-9. (in Russia)

7. E.S. Belousova, A.M.A. Mohamed, M.S.Kh. Al-Mahdawi, A.M. Prudnik Flexible electromagnetic radiation shields based on carbon-containing glued compounds Doklady BGUIR. 2017, Vol. 110, No. 8, pp. 73-78.

8. Yakimov A.N., Andreev P.G., Knyazev V.V. Modeling the propagation of electromagnetic waves in a room, taking into account the influence of local objects / "MAGAZINE OF RADIO ELECTRONICS" N 2, 2015, p. 8 (in Russia)

9. Grundlagen der Radartechnik https://www.radartutorial.eu/

10. Legkiy, N.M., Sabirov, T.R. Advanced Phased Array Antenna (PAA) Research Journal of Applied Sciences 11(11), pp. 1313-1316, 2016

11. Legkiy N.M., Unchenko I.V. Formation of the direction diagram in phased antenna array. Rossiyskiy tekhnologicheskiy zhurnal (Russian Technological Journal). 2019; 7(2): pp. 29-38. (in Russ.).

12. Voskresensky D.I., Gostyukhin V.L., Maksimov V.M., Ponomarev L.I. Microwave Devices and Antennas / Ed. prof. DI. Voskresensky. Ed. 3rd Radio engineering Moscow 2008 (in Russia) 